\title{
Ultra-Widefield Retina Imaging: Principles of Technology and Clinical Applications
}

\author{
이준엽, 사공민 \\ Junyeop Lee, Min Sagong
}

영남대학교 의과대학 안과학교실

Department of Ophthalmology, Yeungnam University College of Medicine, Daegu, Korea

\begin{abstract}
The peripheral retina is frequently affected by various diseases and ultra-widefield (UWF) imaging has become an essential tool for evaluation of peripheral retinal pathology. The wide angle and high resolution of this imaging device were recently updated along with multimodal capabilities, including pseudocolor fundus photographs, fluorescein and indocyanine green angiography, and autofluorescence images. Using this UWF technology, important clinical observations have been made in the normal population and patients with certain diseases including diabetic retinopathy, retinal vascular occlusion, retinal detachment, uveitis, age-related macular degeneration, and choroidal tumors. Although peripheral distortion and the need for clinical validation are obstacles to overcome for the accurate quantification and more generalized use, UWF imaging has provided new insights into a variety of disorders and has become the standard-ofcare imaging modality for many diseases. Therefore, use of UWF retinal imaging for the daily retina practice in the near future is inevitable. This review will describe the principles of UWF technologies with several modalities, and include its clinical applications and then suggest future directions.
\end{abstract}

Keywords: Optomap; Optos; Panoramic imaging; Ultra-widefield; Widefield

\section{Introduction}

The peripheral retina is frequently affected by several diseases including diabetic retinopathy, retinal vascular occlusions, uveitis, degeneration, retinal breaks and detachments. Conventional fundus imaging cannot precisely capture the peripheral lesion that was clinically examined through the wide angle optical lenses. Recent advances in the development of wide field imaging technique have had a significant impact in better assessment of the retinal periphery. Ultra-Widefield (UWF) imaging is one of the advanced technologies. While the conventional fundus imaging modalities are restricted to the central $30^{\circ}$ to $60^{\circ}$, UWF imaging devices facilitate the acquisition of $200^{\circ}$ of the retina in a single image. With a variety of modalities, including pseudocolor images, fundus autofluorescence (FAF), fluorescein angiography (FA), and

\section{Address reprint requests to Min Sagong, MD, PhD}

Department of Ophthalmology, Yeungnam University College of Medicine, \#170 Hyeonchung-ro, Nam-gu, Daegu 42415, Korea

Tel: 82-53-620-3443, Fax: 82-53-626-5936

E-mail: msagong@ynu.ac.kr
Received: 2016. 4. 24

Revised: 2016. 4. 30

Accepted: 2016. 5. 2 
indocyanine green angiography (ICGA), UWF provides additional information for better understanding of retinal and choroidal pathology. This review will describe the principles of UWF technologies with several modalities, including its clinical applications, and then suggest future directions.

\section{Principles of ultra-widefield imaging technology}

\section{Need for development of ultra-widefield imaging}

Conventional fundus imaging modalities use full-field flash illumination and provide a limited view of the posterior pole. Historically, the first fundus camera, introduced by Carl Zeiss and J.W. Nordensen, provided a $20^{\circ}$ fundus image [1]. Several years later, the field of view was expanded to $30^{\circ}$, which became the standard for conventional fundus photography [1]. It can be used for imaging of the optic nerve head and the posterior pole, thus it has been widely used for glaucoma and macular diseases. However, it provided a limited view of the retinal periphery. To overcome this limitation, several protocols using a systemic method or panoramic fundus photography techniques were introduced for visualizing peripheral retinal diseases. The original and modified seven-standard field color fundus photography protocols from the Early Treatment Diabetic Retinopathy Study enable coverage of the mid-peripheral retina to the equator, approximately $75^{\circ}$ [2]. This composite imaging method was extended to nine-standard photography for cytomegalovirus retinitis and acquired immune deficiency syndrome retinopathy [3]. The first panoramic fundus pictures were made from a montage of 19 photographs using a movable fixation light to enable the acquisition of $96^{\circ}$ fundus images [4]. Contact lens-based systems, including Pomerantzeff camera [5], Panoret-1000 [6], RetCam [7], and Staurenghi lens [8], provide up to $160^{\circ}$ panoramic photographs. However, these methods require exact patient alignment, good pupillary dilation, and a skilled photographer, thus their use is impractical in clinical practice. In addition, a single wide field image rather than the panoramic montage has advantages for applications such as FA and ICGA.

\section{Optos ultra-widefield fundus camera module}

The Optos fundus camera (Optos PLC, Dunfermline, UK), a confocal scanning laser ophthalmoscope (SLO)-based UWF imaging system, produces up to $200^{\circ}$ view of the retina, approximately $82 \%$ of the surface area $[9,10]$. The Optos technology utilizes an ellipsoidal mirror to obtain images of the retinal periphery with one capture without the need for bright illumination lighting or a contact lens, and in some patients, pupillary dilation. Since the first commercially available Optos camera (Optos PLC) became available in 2000 , it has been upgraded by addition of multiple laser sources. The initial system was built with $532 \mathrm{~nm}$ and 633 $\mathrm{nm}$ lasers for red and green two-color images, and a $488 \mathrm{~nm}$ blue laser for FA and $805 \mathrm{~nm}$ infrared laser for ICGA were added later.

The SLO contains optical elements including beam-splitters and a confocal aperture. Oscillating galvanometer mirrors provide rapid two-dimensional raster scanning onto an ellipsoidal mirror (Fig. 1). To correct the wavefront aberrations, one focal point of the ellipsoidal mirror lies at the pupillary plane, which enables a wide scanning angle even without pupillary dilation. The compensated light from the retina is focused through a confocal aperture and various filters, and then detected with the image detector. This ellipsoidal mirror-based confocal SLO (cSLO) system enables the wide depth-of-focus and thus in-focus imaging from the anterior retina to the posterior pole. A single scan of a two-color image can take only 0.25 seconds to provide approximately 20-pixel resolution per degree [11].

\section{Stereographic projection software to correct peripheral distortion}

One of the critical limitations from a broad viewing angle

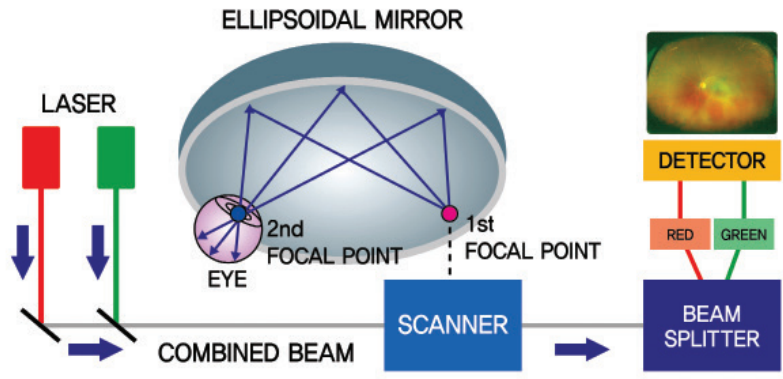

Figure 1. Schematic illustration of ultra-widefield fundus camera module using an ellipsoidal mirror. Scanning laser light is focused through the first focal point while the patient positions his eye coincident with the second focal point, which produces a wide scanning angle and peripheral fundus examination even without pupillary dilation. 
using the ellipsoidal mirror was distortion of peripheral retina. The most peripheral areas magnified the posterior pole up to 2 times, and the horizontal axis was stretched compared with the vertical axis $[12,13]$. However, recent advances in UWF imaging hardware and software have corrected peripheral distortion and enabled accurate quantification of the peripheral retina [14]. Using stereographic projection software, now available in commercial UWF devices, images can be obtained at different gaze angles to be montaged and corrected for peripheral distortion [15]. Sagong et al. [14] validated the precision and accuracy of the measurements derived from this software in eyes containing prosthetic implants of known sizes. Therefore, using this software, researchers can calculate the anatomically correct areas from UWF images in metric units.

\section{Ultra-widefield imaging modalities}

The red and green laser wavelength in the Optos system provides two-color images of the retina. The blue laser is not available in the current Optos system. The green laser highlights the retina and its vessels, while the red laser highlights deeper tissues. These two laser components can be operated simultaneously for rapid attainment of pseudocolor images. FAF imaging is also available in the Optos system. Autofluorescence from lipofuscin is excited by $532 \mathrm{~nm}$ laser and filtered by $570 \mathrm{~nm}$ to $780 \mathrm{~nm}$ emission filter in the Optos system. Compared to the short-wavelength or near-infrared FAF in the Heidelberg HRA cSLO (Heidelberg Engineering, Heidelberg, Germany), the excitation from the $532 \mathrm{~nm}$ green laser has an advantage in that it is less affected by the absorbance from nuclear sclerotic cataracts [16]. Ultra-widefield FA is available in the Optos system since the P200A model provides a high-resolution wide-angle angiographic module. Single capture using a 488-nm laser with a 500-nm barrier filter enables angiographic view of peripheral vascular structure, including perfusion status, leakage, and staining, which can be missed on the seven-standard fields of the Early Treatment Diabetic Retinopathy Study [17-20]. ICGA was recently added to the multimodal capabilities of the Optos. ICGA is performed using an $805 \mathrm{~nm}$ excitation beam and an $835 \mathrm{~nm}$ barrier filter. The diagnostic ability of UWF ICGA has been demonstrated in several studies in a variety of chorioretinal diseases $[21,22]$. In addition to the wide panoramic image of the fundus, the high-density raster scanning of the Optos can provide macular details, comparable with the Hei- delberg HRA cSLO system [21].

\section{Clinical applications of ultra-widefield imaging}

\section{Diabetic retinopathy}

Although diabetic retinopathy (DR) is an ischemic vascular disease frequently affecting the peripheral retina, methods for assessing and quantifying the retinal periphery in DR were limited. The potential for use of UWF imaging, particularly UWF FA, for enhancing diagnosis and treatment in DR has been demonstrated [23]. Several studies compared UWF pseudocolor images with conventional fundus photographs in the screening of DR [24-27]. Wilson et al. [26] reported $83.6 \%$ sensitivity for UWF images versus $82.9 \%$ for digital photography in identifying DR. Silva et al. [25] found that UWF matched 7 standard grading of severity in $80 \%$ of eyes and was within 1 level in $94.5 \%$ of eyes. In $10 \%$ of eyes, the additional peripheral findings identified by UWF suggested a more severe level of retinopathy than the seven-standard fields. Collectively, these studies demonstrated that UWF can be a better screening tool for DR. There is an ongoing DRCRnet protocol determining whether evaluation of the retinal periphery with UWF images can improve our ability to assess and predict worsening of DR compared with standard photography [28].

Fluorescein angiography shows retinal microvascular changes that are undetectable in fundus examination, and is therefore an essential diagnostic tool for DR. Conventional FA displays $30^{\circ}$ field of the retina, thus missing the peripheral retina. With the UWF FA, up to $200^{\circ}$ of the retina can be viewed as a single photograph, providing a more comprehensive assessment of the extent of a retinal disease process (Fig. 2). Multiple studies in DR have reported that UWF FA was superior to the seven-standard fields in demonstrating angiographic abnormalities [17,29,30]. Wessel et al. [30] reported that UWF FA displayed 3.9 times more nonperfusion, 1.9 times more neovascularization, and 3.8 times more panretinal photocoagulation compared with a simulated 7-field overlay. In addition, the seven-standard fields would have missed pathology shown on UWF FA images in $10 \%$ of eyes [30]. Other studies have demonstrated the association between peripheral retinal nonperfusion and the occurrence of neovascularization, macular ischemia, and diabetic mac- 

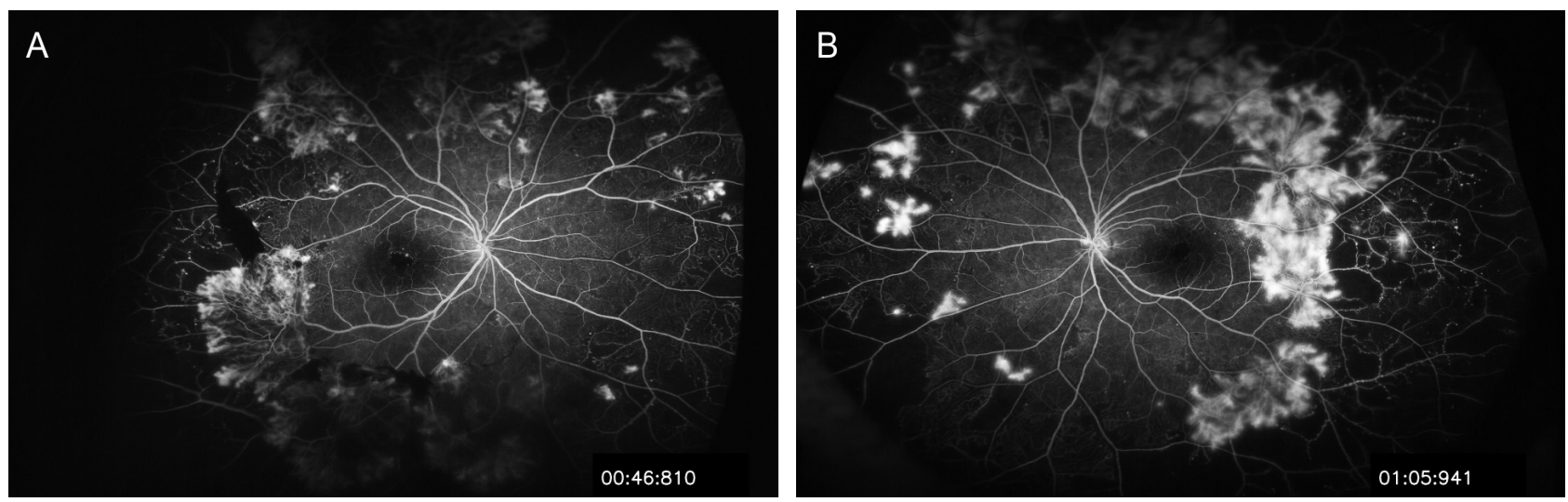

Figure 2. Ultra-widefield fluorescein angiography of proliferative diabetic retinopathy. Images of right (A) and left (B) eyes of a 42-year-old patient with type 1 diabetes show microaneurysm, venous beading, extensive neovascularization elsewhere, and peripheral capillary nonperfusion.

ular edema (DME) [20,31-33]. Another study suggested that UWF FA will be used to guide the management of DR [34]. UWF-guided targeted retinal photocoagulation to areas of nonperfusion with successful regression of retinal neovascularization. Therefore, UWF FA can detect peripheral pathologies, which may alter treatment plans and provide new insights into the pathogenesis of DR and DME.

\section{Retinal vascular occlusion}

Retinal vascular occlusive diseases are common indications for UWF imaging. They are similar to DR in that it is associated with nonperfusion, macular edema, and neovascularization. Prasad et al. [19], who evaluated the use of UWF FA for study of the peripheral angiographic features of branch retinal vein occlusions and hemicentral retinal vein occlusions, found that areas of angiographic nonperfusion peripheral to the equator were significantly associated with macular edema and neovascularization. They suggested that areas of untreated retinal nonperfusion may be the source of production of biochemical mediators that promote $\mathrm{NV}$ and macular edema. Tsui et al. [35] used an ischemic index in retinal vascular occlusions that describes a ratio of nonperfused retina over the whole retinal area measured manually from UWF FA. Singer et al. [36] reported that a higher ischemic index on UWF FA images correlated with greater macular thickness and worse vision in retinal vascular occlusion. Eyes with a higher ischemic index showed a better gain in visual acuity and greater decrease in retinal thickness on optical coherence tomography (OCT) after treatment [36]. Therefore, UWF imaging provides more detailed characteristics of vein occlusions in terms of perfusion or ischemic status throughout the whole retinal area, which could be an important factor determining the prognosis after treatment.

\section{Retinal detachment}

UWF pseudocolor and autofluorescence imaging show abnormalities in the rhegmatogenous retinal detachment allowing excellent localization of causative retinal breaks and demarcation of the extent of the retinal detachment [37]. Compared to indirect ophthalmoscopy, UWF imaging can, in some cases, provide a more precise documentation of the extent of the detachment [38]. The UWF FAF provides prognostic information and a means for examining the postoperative functional status of the previously detached retina [37,39]. Witmer et al. [37] reported that patients with macula-involving detachments who showed persistent postoperative granular autofluorescent changes had significantly worse preoperative visual acuity and showed a trend toward worse postoperative visual prognosis. Labriola et al. [40] reported on a case in which UWF and UWF FA were helpful in documenting neovascularization and macrocyst formation associated with chronic retinal detachment (Fig. 3). The value of UWF imaging lies in its use as an adjunct to clinical examination for documenting the retinal detachment and providing functional information about postoperative prognosis.

\section{Pediatric retina and retinopathy of prematurity}

The RetCam has provided a wide-field fundus image with fluorescein angiography capabilities in pediatric patients, however, use of this contact-based system requires a patient's cooperation or administration of general anesthesia. 

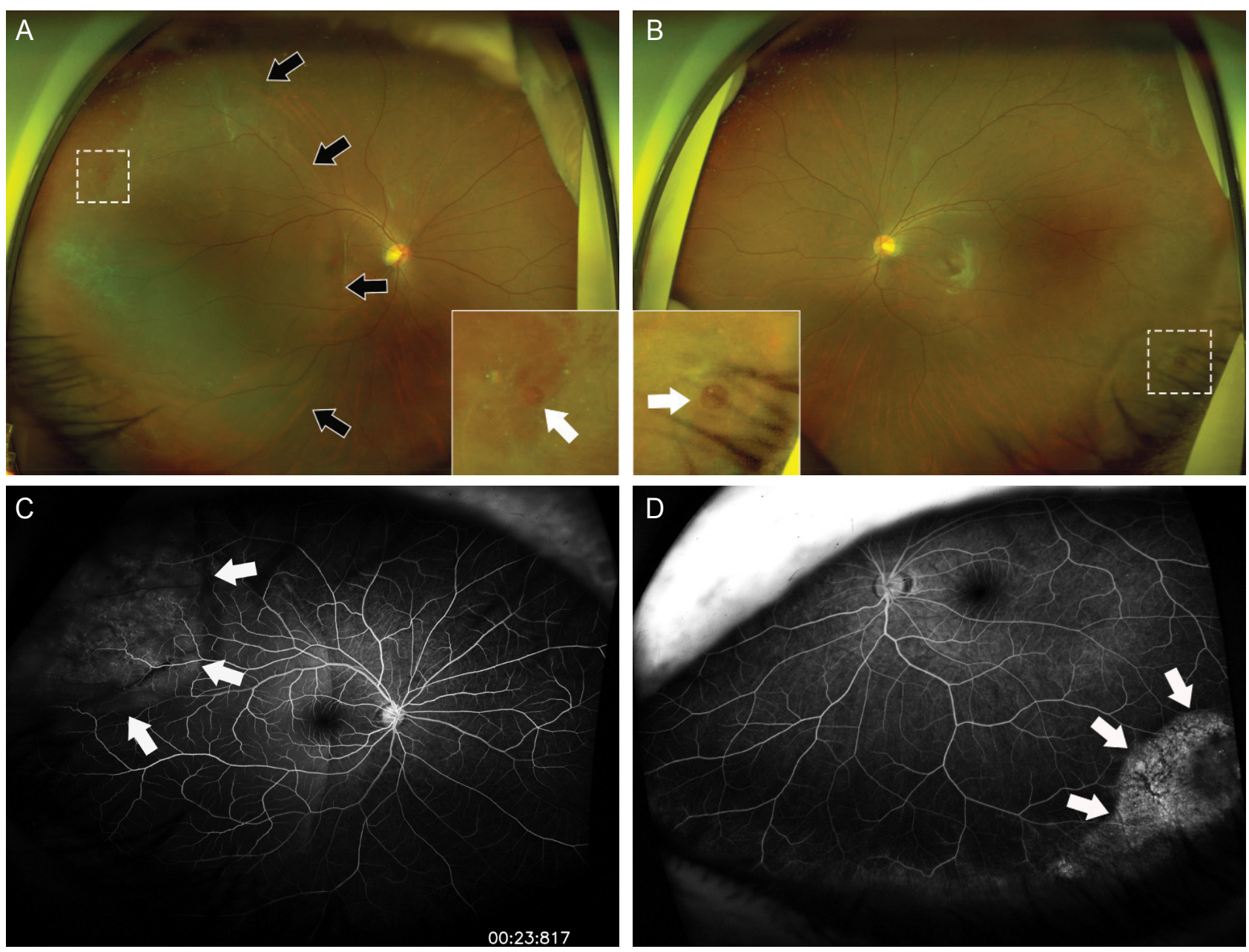

Figure 3. Ultra-widefield documentation of rhegmatogenous retinal detachment. Two pseudocolor images (A, B) and fluorescein angiography (C, D) of 20-year-old patient diagnosed as rhegmatogenous retinal detachment in right eye. (A) Small round retinal breaks (inset, white arrow) and extent of retinal detachment (black arrows) were evident in the affected eye. (B) Lattice degeneration and a small round break (inset, white arrow) were screened in the fellow eye. (C, D) Fluorescein angiography provided additional precise documentation on the margin of hyperfluorescence suggesting chronic retinal detachment (white arrows) in the affected eye (C) and the fellow eye (D).

Optos UWF imaging can be an alternative method for documenting pediatric retinal pathology in an outpatient setting. Tsui et al. [41] demonstrated the utility of UWF imaging for a pediatric retina in a group of children 5 years to 12 years old. UWF FA images showed clear views of the different stages of retinopathy of prematurity (ROP) features at the posterior pole and peripheral retina. With the help of UWF FA images, regression of ROP features was identified, following laser and intravitreal bevacizumab treatment $[42,43]$. In addition, the "skip areas" that were missed by initial laser treatment could be identified in the peripheral retina and managed accordingly [43]. In these patients, luorescein angiography can be performed using oral fluorescein, providing a less invasive outpatient option [44]. In the Coat's disease, an idiopathic retinal vascular abnormality of young males, UWF FA can be used successfully as an outpatient procedure in the pediatric patient population and used in targeting laser therapy without the necessity of anesthesia [45].

\section{Age-related macular degeneration}

There might be controversy regarding the use of UWF images for macular disease. In cases of peripheral exudative chorioretinopathy, UWF FA could be of help in identifying the peripheral lesion. However, those cases are unusual in typical age-related macular degeneration (AMD). Nevertheless, pseudocolor images of Optos can provide an image 
including macular details, comparable with the Heidelberg Retina Angiograph (HRA) cSLO system [21]. In the Reykjavik Eye Study, UWF imaging showed $96 \%$ agreement with conventional $45^{\circ}$ images when grading patients with drusen, geographic atrophy, and choroidal neovascularization [46]. In addition, the UWF ICGA can delineate the choroidal vessel of the macula and periphery at high resolution [21]. UWF ICGA may provide information on peripheral choroidal vascular changes associated with macular choroidopathy in
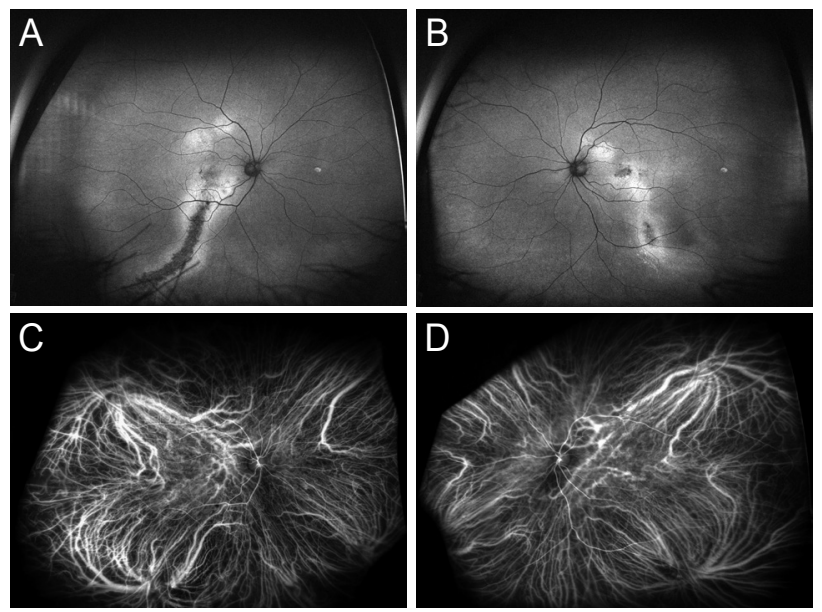

Figure 4. Ultra-widefield autofluorescene and indocyanine green angiography of central serous chorioretinopathy. Images of right (A) and left (B) eyes of a 58-year-old patient referred for an evaluation of macular degeneration. Gravitational tracks in both eyes support the diagnosis of chronic central serous chorioretinopathy. (C, D) Indocyanine green angiography reveals dilated and irregular choroidal vessels in the macula in both eyes.
AMD or polypoidal choroidal vasculopathy. Several studies have demonstrated autofluorescence characteristics of peripheral retinal in AMD. Heussen et al. [47] reported that $70 \%$ of eyes with AMD had peripheral abnormalities detectable on UWF FAF and pseudocolor. In a large prospective study abnormal peripheral FAF patterns were more frequent in eyes with neovascular AMD compared to eyes with non-neovascular AMD [48]. With the combination of strong fellow eye concordance, they suggested that peripheral abnormalities could be used as biomarkers for grading disease severity or even predicting an individual's risk of neovascular AMD.

\section{Central serous chorioretinopathy}

Central serous chorioretinopathy (CSC), a maculopathy that causes serous detachments secondary to choroidal hyperpermeability and retinal pigment epithelium (RPE) leaks, is believed to mainly involve the center of the macula. Recent studies using UWF AF and UWF ICGA demonstrated that the extent of CSC frequently involves the peripheral retina (Fig. 4) [22], and identified peripheral choroidal outflow congestion as a possible contributing factor to the pathogenesis of CSC. Dilated choroidal vessels detected in UWF ICGA may serve as a predictor for disease progression. A new method for subtracting and binarizing UWF angiographic images and quantifying the choroidal vessel density was recently developed [49]. The choroidal vessel density increased in eyes with CSC compared to control eyes.
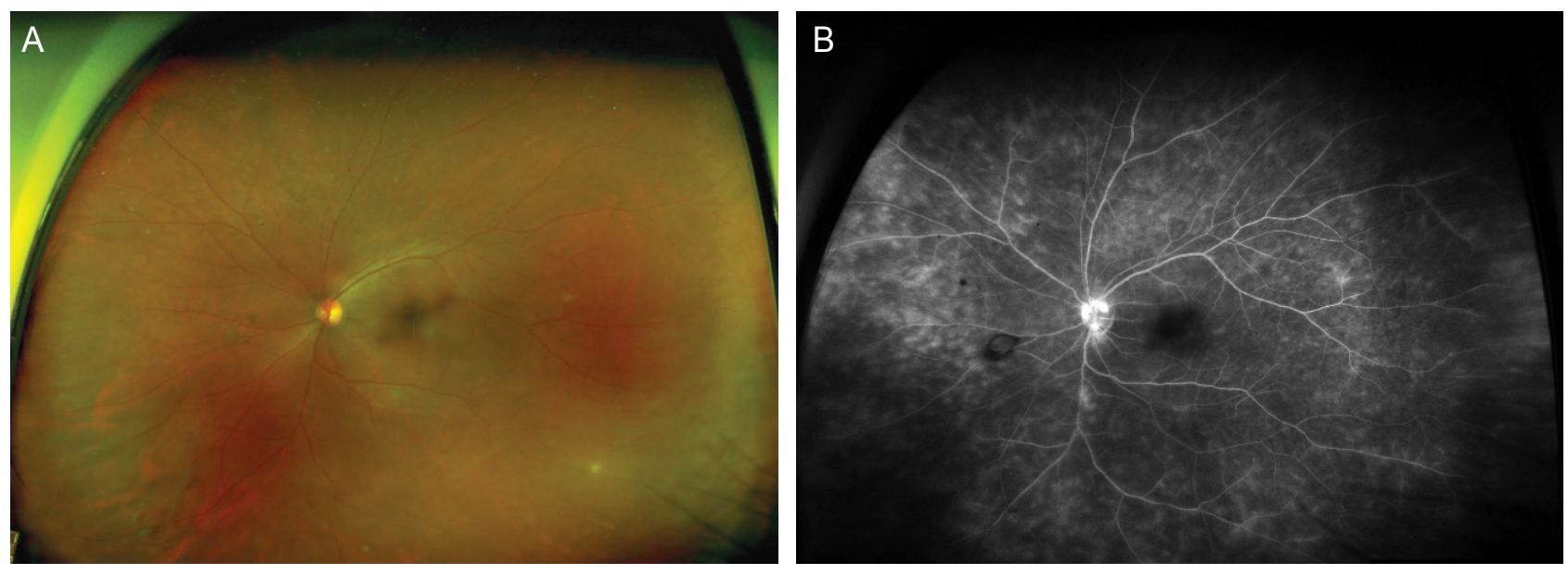

Figure 5. Ultra-widefield fluorescein angiography of uveitis. A pseudocolor image (A) and fluorescein angiography (B) of 38-year-old patient with Bechet's disease. Although the pseudocolor image displayed mild vitreous opacity, fluorescein angiography demonstrated profound vascular leakage suggesting active inflammation, which influenced the decision of management. 


\section{Uveitis and retinal vasculitis}

The diagnosis of intermediate uveitis or retinal vasculitis was challenging in the conventional $30^{\circ}$ to $60^{\circ}$ fluorescein angiography. Decisions regarding the management of uveitis depend on the clinical findings and angiographic pattern of the inflammatory lesions. Kaines et al. [50] reported that UWF images enabled clear identification of peripheral retinal lesions and greatly enhanced objective documentation of disease activity and progression. Similar to DR, UWF imaging enabled clear visualization of the areas of neovascularization and peripheral nonperfusion, which requires sector photocoagulation, hence minimizing complications. Compared to 9-field standard FA, UWF FA found significantly more leakage even in areas captured well with standard angiography such as the posterior pole in uveitis [51]. In addition, UWF FAF is useful in determining whether lesions are in a state of active inflammation in patients with uveitis. Mesquida et al. [52], who assessed the role of UWF imaging in the diagnosis and management of retinal vasculitis associated with Behçet disease, reported that UWF two color images enabled clear documentation of peripheral vein sheathing and retinal infiltrates and greatly simplified longitudinal comparisons for disease activity and progression (Fig. 5). In addition, UWF FAF displayed multiple hyperfluorescent spots in the retinal periphery, suggesting that active vasculitis may induce pigment alterations in the retinal periphery [52]. In addition, UWF images have been used as a grading system for the grading of severity in the sunset glow fundus of Vogt-Koyanagi-Harada disease, and for detection of more peripheral lesions in patients with cytomegalovirus retinitis $[53,54]$.

\section{Retinal and choroidal dystrophies}

UWF FAF imaging has an advantage in that it can detect the subtle change of RPE from periphery in retinal choroidal dystrophies. UWF FA provides additional information on capillary loss. Yuan et al. [55] reported on several patients with choroideremia and gyrate atrophy. Using the UWF FAF, Oishi et al. [56] classified retinitis pigmentosa (RP) based on the pattern of autofluorescence and demonstrated its correlation with visual acuity and mean deviation on the Humphrey visual field test. Ogura et al. [57] found a close agreement between the spatial extents of hypoautofluorescence with the degree of visual field loss in RP. Therefore, the combination of UWF FAF, visual field deficits, and elect- roretinography might become a standard multimodal test for monitoring chorioretinal dystrophies.

\section{Choroidal tumors}

Choroidal melanoma, the most common primary malignant tumor, metastasizes into several organs. Detection of the primary choroidal melanoma in the early phase is important because metastasis may occur before the primary tumor is diagnosed. Reznicek et al. [58] reported that the mean fundus autofluorescence intensity of melanomas was significantly lower than of the autofluorescence of benign choroidal nevi. In combination with previous clinical criteria, findings of UWF FAF may be helpful in the differential diagnosis. In addition, measurements of choroidal lesion size on UWF images correlate reasonably well with ultrasound measurements [59]. In addition, UWF FA is crucial for the identification and monitoring of retinal hemangioblastomas in von Hippel-Lindau disease [60]. The utility of UWF ICGA in choroidal tumors will be evaluated in further studies.

\section{Future directions}

In imaging of the peripheral retina, numerous technological improvements have been made over the last 10 years, and it has been used effectively and increasingly in clinical settings. The technology of UWF has become important clinically with regards to early diagnosis and monitoring of many retinal diseases and may have significant utility for use in clinical or basic research. Applicability of UWF imaging will be expanded when it is combined with other updated imaging modalities. Of note, UWF steering-based spectral domain optical coherence tomography (SD-OCT) images of the retinal periphery have already been reported [61]. This method enabled unprecedented acquisition of high resolution cross-sectional structural information on a variety of peripheral retinal entities and pathologies. If this combination of UWF and SD-OCT is upgraded, high resolution of whole retinal tissue could be rendered as a 3 dimensional structure, adding a thickness profile to the UWF information. For the general use of UWF imaging as a screening tool for various retinal diseases, several obstacles remain due to the high cost of the equipment, which is not easily transportable. Nevertheless, the future of UWF imaging will definitely include wider clinical applications and expendability. Substantial 
research is ongoing to validate and expand the utilization of UWF imaging. In the near future, UWF imaging technology will be essential for the daily retina practice.

\section{Conflicts of interest}

There are no conflicts of interest.

\section{References}

1. Ciardella A BD. Wide field imaging. In: Agarwal A, ed. Fundus fluorescein and indocyanine green angiography: a textbook and atlas, 1st ed. New York: Slack Inc., 2007;chap. 11.

2. Diabetic retinopathy study. Report Number 6 . Design, methods, and baseline results. Report Number 7. A modification of the Airlie House classification of diabetic retinopathy. Prepared by the Diabetic Retinopathy. Invest Ophthalmol Vis Sci 1981;21(1 Pt 2):1-226.

3. Clark TM. Retinal photography and angiography via film and digital imaging techniques. In: Arevalo JF, ed. Retinal angiography and optical coherence tomography, 1st ed. New York: Springer, 2008; chap. 1.

4. Lotmar W. A fixation lamp for panoramic fundus pictures. Klin Monbl Augenheilkd 1977;170:767-74.

5. Pomerantzeff O. Equator-plus camera. Invest Ophthalmol 1975;14:401-6.

6. Pe'er J, Sancho C, Cantu J, et al. Measurement of choroidal melanoma basal diameter by wide-angle digital fundus camera: a comparison with ultrasound measurement. Ophthalmologica 2006:220:194-7.

7. Schwartz SD, Harrison SA, Ferrone PJ, Trese MT. Telemedical evaluation and management of retinopathy of prematurity using a fiberoptic digital fundus camera. Ophthalmology 2000;107:25-8.

8. Staurenghi G, Viola F, Mainster MA, et al. Scanning laser ophthalmoscopy and angiography with a wide-field contact lens system. Arch Ophthalmol 2005;123:244-52.

9. Shoughy SS, Arevalo JF, Kozak I. Update on wide- and ultra-widefield retinal imaging. Indian J Ophthalmol 2015;63:57581.

10. Nagiel A, Lalane RA, Sadda SR, Schwartz SD. Ultra-widefield fundus imaging: a review of clinical applications and future trends. Retina 2016;36:660-78.

11. Seidensticker F, Neubauer AS, Wasfy T, et al. Wide-field fundus autofluorescence corresponds to visual fields in chorioretinitis patients. Clin Ophthalmol 2011;5:1667-71.
12. Croft DE, van Hemert J, Wykoff CC, et al. Precise montaging and metric quantification of retinal surface area from ultra-widefield fundus photography and fluorescein angiography. Ophthalmic Surg Lasers Imaging Retina 2014;45:312-7.

13. Oishi A, Hidaka J, Yoshimura N. Quantification of the Image Obtained With a Wide-Field Scanning Ophthalmoscope. Invest Ophthalmol Vis Sci 2014;55:2424-31.

14. Sagong M, van Hemert J, Olmos de Koo LC, et al. Assessment of accuracy and precision of quantification of ultra-widefield images. Ophthalmology 2015;122:864-6.

15. Singer $M$, Sagong $M$, van Hemert J, et al. Ultra-widefield imaging of the peripheral retinal vasculature in normal subjects. Ophthalmology 2016;123:1053-9.

16. Spaide RF. Fundus autofluorescence and age-related macular degeneration. Ophthalmology 2003;110:392-9.

17. Friberg TR, Gupta A, Yu J, et al. Ultrawide angle fluorescein angiographic imaging: a comparison to conventional digital acquisition systems. Ophthalmic Surg Lasers Imaging Retina 2008:39:304-11.

18. Manivannan A, Plskova J, Farrow A, et al. Ultra-wide-field fluorescein angiography of the ocular fundus. Am J Ophthalmol 2005;140:525-7.

19. Prasad PS, Oliver SC, Coffee RE, et al. Ultra wide-field angiographic characteristics of branch retinal and hemicentral retinal vein occlusion. Ophthalmology 2010;117:780-4.

20. Oliver SC, Schwartz SD. Peripheral vessel leakage (PVL): a new angiographic finding in diabetic retinopathy identified with ultra wide-field fluorescein angiography. Semin Ophthalmol 2010;25:27-33.

21. Klufas MA, Yannuzzi NA, Pang CE, et al. Feasibility and clinical utility of ultra-widefield indocyanine green angiography. Retina 2015;35:508-20.

22. Pang CE, Shah VP, Sarraf D, Freund KB. Ultra-widefield imaging with autofluorescence and indocyanine green angiography in central serous chorioretinopathy. Am J Ophthalmol 2014;158:362-71.e2

23. Tan CS, Sadda SR, Hariprasad SM. Ultra-widefield retinal imaging in the management of diabetic eye diseases. Ophthalmic Surg Lasers Imaging Retina 2014;45:363-6.

24. Neubauer AS, Kernt M, Haritoglou C, et al. Nonmydriatic screening for diabetic retinopathy by ultra-widefield scanning laser ophthalmoscopy (Optomap). Graefes Arch Clin Exp Ophthalmol 2008;246:229-35

25. Silva PS, Cavallerano JD, Sun JK, et al. Peripheral lesions identified by mydriatic ultrawide field imaging: distribution and po- 
tential impact on diabetic retinopathy severity. Ophthalmology 2013;120:2587-95.

26. Wilson PJ, Ellis JD, MacEwen CJ, et al. Screening for diabetic retinopathy: a comparative trial of photography and scanning laser ophthalmoscopy. Ophthalmologica 2010;224:251-7.

27. Kernt M, Hadi I, Pinter F, et al. Assessment of diabetic retinopathy using nonmydriatic ultra-widefield scanning laser ophthalmoscopy (Optomap) compared with ETDRS 7-field stereo photography. Diabetes Care 2012;35:2459-63.

28. Jaeb Center for Health Research. Peripheral Diabetic Retinopathy (DR) Lesions on Ultrawide-field Fundus Images and Risk of DR Worsening Over Time [Internet]. Diabetic Retinopathy Clinical Research Network (DRCRnet) public web site; 2015 [cited 2016 April 20]. Available from: http://drcrnet.jaeb.org/Studies. aspx?RecID=239.

29. Kong M, Lee MY, Ham DI. Ultrawide-field fluorescein angiography for evaluation of diabetic retinopathy. Korean J Ophthalmol 2012;26:428-31.

30. Wessel MM, Aaker GD, Parlitsis G, et al. Ultra-wide-field angiography improves the detection and classification of diabetic retinopathy. Retina 2012;32:785-91.

31. Patel RD, Messner LV, Teitelbaum B, et al. Characterization of ischemic index using ultra-widefield fluorescein angiography in patients with focal and diffuse recalcitrant diabetic macular edema. Am J Ophthalmol 2013;155:1038-44.e2.

32. Wessel MM, Nair N, Aaker GD, et al. Peripheral retinal ischaemia, as evaluated by ultra-widefield fluorescein angiography, is associated with diabetic macular oedema. Br J Ophthalmol 2012;96:694-8.

33. Sim DA, Keane PA, Rajendram R, et al. Patterns of peripheral retinal and central macula ischemia in diabetic retinopathy as evaluated by ultra-widefield fluorescein angiography. Am J Ophthalmol 2014;158:144-53.e1.

34. Muqit MM, Marcellino GR, Henson DB, et al. Optos-guided pattern scan laser (Pascal)-targeted retinal photocoagulation in proliferative diabetic retinopathy. Acta ophthalmol 2013;91:2518.

35. Tsui I, Kaines A, Havunjian MA, et al. Ischemic index and neovascularization in central retinal vein occlusion. Retina 2011;31:10510.

36. Singer M, Tan CS, Bell D, Sadda SR. Area of peripheral retinal nonperfusion and treatment response in branch and central retinal vein occlusion. Retina 2014;34:1736-42.

37. Witmer MT, Cho M, Favarone G, et al. Ultra-wide-field autofluorescence imaging in non-traumatic rhegmatogenous retinal detachment. Eye (Lond) 2012;26:1209-16

38. Kornberg DL, Klufas MA, Yannuzzi NA, et al. Clinical utility of ultra-widefield imaging with the Optos Optomap compared with indirect ophthalmoscopy in the setting of non-traumatic rhegmatogenous retinal detachment. Semin Ophthalmol 2015;21:1-8.

39. Salvanos P, Navaratnam J, Ma J, et al. Ultra-widefield autofluorescence imaging in the evaluation of scleral buckling surgery for retinal detachment. Retina 2013:33:1421-7.

40. Labriola LT, Brant AM, Eller AW. Chronic retinal detachment with secondary retinal macrocyst and peripheral neovascularization. Semin Ophthalmol 2009;24:2-4.

41. Tsui I, Franco-Cardenas V, Hubschman JP, Schwartz SD. Pediatric retinal conditions imaged by ultra wide field fluorescein angiography. Ophthalmic Surg Lasers Imaging Retina 2013;44:59-67.

42. Shroff D, Narain S, Gupta C, et al. Non-contact ultra-widefield imaging in lasered retinopathy of prematurity. Indian J Pediatr 2015 Dec 17. [Epub ahead of print]

43. Patel CK, Fung TH, Muqit MM, et al. Non-contact ultra-widefield imaging of retinopathy of prematurity using the Optos dual wavelength scanning laser ophthalmoscope. Eye (Lond) 2013;27:589-96.

44. Fung TH, Muqit MM, Mordant DJ, et al. Noncontact high-resolution ultra-wide-field oral fluorescein angiography in premature infants with retinopathy of prematurity. JAMA ophthalmol 2014;132:108-10.

45. Kang KB, Wessel MM, Tong J, et al. Ultra-widefield imaging for the management of pediatric retinal diseases. J Pediatr Ophthalmol Strabismus 2013;50:282-8.

46. Csutak A, Lengyel I, Jonasson F, et al. Agreement between image grading of conventional (45 degrees) and ultra wide-angle (200 degrees) digital images in the macula in the Reykjavik eye study. Eye (Lond) 2010;24:1568-75.

47. Heussen FM, Tan CS, Sadda SR. Prevalence of peripheral abnormalities on ultra-widefield greenlight (532 nm) autofluorescence imaging at a tertiary care center. Invest Ophthalmol Vis Sci 2012;53:6526-31.

48. Tan CS, Heussen F, Sadda SR. Peripheral autofluorescence and clinical findings in neovascular and non-neovascular age-related macular degeneration. Ophthalmology 2013;120:1271-7.

49. Hirahara S, Yasukawa T, Kominami A, et al. Densitometry of choroidal vessels in eyes with and without central serous chorioretinopathy by wide-field indocyanine green angiography. Am J Ophthalmol 2016. [Epub ahead of print]

50. Kaines A, Tsui I, Sarraf D, Schwartz S. The use of ultra wide field fluorescein angiography in evaluation and management of 
uveitis. Semin Ophthalmol 2009;24:19-24.

51. Nicholson BP, Nigam D, Miller D, et al. Comparison of wide-field fluorescein angiography and 9-field montage angiography in uveitis. Am J Ophthalmol 2014;157:673-7.

52. Mesquida M, Llorenç V, Fontenla JR, et al. Use of ultra-wide-field retinal imaging in the management of active Behçet retinal vasculitis. Retina 2014;34:2121-7.

53. Lee EK, Lee SY, Yu HG. A clinical grading system based on ultra-wide field retinal imaging for sunset glow fundus in Vogt-Koyanagi-Harada disease. Graefes Arch Clin Exp Ophthalmol 2015;253:359-68.

54. Mudvari SS, Virasch VV, Singa RM, MacCumber MW. Ultra-widefield imaging for cytomegalovirus retinitis. Ophthalmic Surg Lasers Imaging 2010;41:311-5.

55. Yuan A, Kaines A, Jain A, et al. Ultra-wide-field and autofluorescence imaging of choroidal dystrophies. Ophthalmic Surg Lasers Imaging 2010;41 Online:e1-5.

56. Oishi A, Ogino K, Makiyama Y, et al. Wide-field fundus autofluorescence imaging of retinitis pigmentosa. Ophthalmology
2013:120:1827-34.

57. Ogura S, Yasukawa T, Kato A, et al. Wide-field fundus autofluorescence imaging to evaluate retinal function in patients with retinitis pigmentosa. Am J Ophthalmol 2014;158:1093-8.

58. Reznicek L, Stumpf C, Seidensticker F, et al. Role of wide -field autofluorescence imaging and scanning laser ophthalmoscopy in differentiation of choroidal pigmented lesions. Int J Ophthalmol 2014;7:697-703.

59. Kernt M, Schaller UC, Stumpf C, et al. Choroidal pigmented lesions imaged by ultra-wide-field scanning laser ophthalmoscopy with two laser wavelengths (Optomap). Clin Ophthalmol 2010;4:829-36.

60. Heimann H, Jmor F, Damato B. Imaging of retinal and choroidal vascular tumours. Eye (Lond) 2013;27:208-16.

61. Choudhry N, Golding J, Manry MW, Rao RC. Ultra-widefield steering-based spectral-domain optical coherence tomography imaging of the retinal periphery. Ophthalmology 2016 Mar 16. pii: S0161-6420(16)00151-2. [Epub ahead of print]. 